\title{
MIGRANTI E RIFUGIATE. ANTROPOLOGIA, GENERE E POLITICA
}

\author{
Migrants and refugees. Anthropology, gender and politics
}

\author{
PINELLI, Barbara. Milano: Raffaello Cortina Editore, 2019, 236p.
}

Emanuela Bini*

Il testo di Barbara Pinelli, docente di Antropologia dei processi migratori presso I'Università degli Studi di Milano-Bicocca, indaga prospettive di genere e di antropologia femminista per descrivere i processi sociali che caratterizzano la mobilità umana, concentrandosi sulle donne rifugiate e migranti.

In contrasto con la concezione che ritrae le donne immigrate come soggetti sospesi nella storia, il testo mostra quanto siano proprio loro stesse a mettere in discussione le strutture di dominio e di prevaricazione presentandosi sulla scena politica come migranti e richiedenti asilo, superando le linee di genere e di classe. Le ricerche etnografiche presentate nel volume sottolineano infatti come nel tempo sia mutata la visione della donna nella migrazione. Negli anni Novanta prevalevano rappresentazioni che consideravano la donna migrante come vittima (oppressa nei Paesi di origine, nello spazio domestico, nel mercato del lavoro dei Paesi d'immigrazione - Hosken, 1981; Fusaschi, 2011) e in seguito come dipendente (dai percorsi migratori maschili e dagli uomini, dalle strutture d'assistenza o dalle economie domestiche - Lyndsay, 1983). Successivamente è emersa la concezione che attribuisce alle donne il ruolo di protagoniste nella migrazione, capaci di emanciparsi dai modelli tradizionali, di essere "significanti privilegiate nella differenza" (Grewal, Kaplan, 1994).

Nel corso degli anni, la percezione delle migranti come vittime da guardare con compassione ha dunque lasciato spazio ad una visione che ne esalta lo spirito d'iniziativa e il coraggio. In particolare, le donne vittime di persecuzioni e discriminazioni esercitando il diritto di asilo per chiedere protezione in un altro Paese si ribellano a soprusi e violenze legate al genere perpetrate nel Paese di origine. Opponendosi a mutilazioni genitali, alla prospettiva di un matrimonio

Laureata in Lingue e Letterature Straniere e in Scienze della Formazione Primaria presso I'Università degli Studi di Bologna. Bologna, Italia. E-mail: emanuela.bini1@istruzione.it. Orcid: 0000-0001-7604-3662. 
forzato, alla violenza domestica e a un ruolo di genere imposto dalla società, ciascuna richiedente asilo porta avanti una lotta contro le iniquità lesive dei diritti umani (Del Guercio, 2018). Pinelli sostiene la valenza politica dell'azione delle richiedenti asilo e delle rifugiate che cercano di scardinare il clima di ingiustizia elevando il proprio corpo - segnato dalla violenza, dal dolore, dalla censura - a mezzo per opporsi al sistema prevaricante (de Laurentis, 1990).

Il libro è articolato in tre capitoli. Nel primo capitolo l'autrice ripercorre la nascita dell'etnografia femminista e il dialogo con le istanze dell'antropologia (Code, 2000; McPherson, 2000; Starr, 2000; Mehta, 2000; Snyder, 2008).

Nel secondo capitolo viene approfondita l'importanza del genere come categoria analitica per lo studio della mobilità umana, aspetto che negli anni Novanta ha portato alla revisione di istanze condivise e universali, quali l'emancipazione delle donne, la libertà di scelta e la modernità (HondagneuSotelo, 1994; Pessar, Mahler, 2003; Donato, Gabaccia, Holdway, Manalansan, Pessar, 2006; Lutz, 2010).

Nel terzo capitolo Pinelli si concentra sulle rifugiate e sulle migrazioni forzate delle donne. Nel corso degli ultimi anni le donne richiedenti asilo e rifugiate sono diventate terreno di profonde rappresentazioni politiche considerando l'intersezione tra "razza", genere e cultura incorporata nelle politiche umanitarie. Contestualmente è emersa la necessità di scardinare I'idea secondo cui l'esperienza delle rifugiate sia riconducibile a una serie di bisogni e diritti "standard". La critica alla visione omologante deriva dalla consapevolezza dell'unicità dell'esperienza di ciascuna rifugiata, caratterizzata da una soggettività sociale e politica che va conosciuta per dare voce agli aspetti critici della diversità (Indra, 1993). Nel capitolo si affronta anche il tema della violenza di genere nelle migrazioni forzate alla luce delle normative internazionali, della riflessione antropologica, della critica postcoloniale e del femminismo giuridico (Salvatici 2004; Pinelli, 2013, 2014, 2017; Rigo, 2016).

Proponendo un'angolatura teorica innovativa, il volume esplora un tema molto complesso e mostra il potenziale politico delle prospettive di genere e femministe nell'indagare i movimenti migratori, le strutture di dominio e le lotte per i diritti umani.

\section{Bibliografia}

CODE, Lorraine. Introduction. In: CODE, Lorraine (a cura di). Encyclopedia of Feminist Theory. London: Routledge, 2000, p. XVI-XXV.

DE LAURENTIS, Teresa. Soggetti eccentrici. Milano: Feltrinelli, 1990.

DEL GUERCIO, Adele. Persecuzione e violenza di genere. Quando sono le donne a chiedere asilo. Rassegna di Diritto Pubblico Europeo, v. XVII, n. 1, p. 151-186, 2018. 
DONATO, Katharine M.; GABACCIA, Donna; HOLDWAY Jennifer; MANALANSAN, Martin IV; PESSAR, Patricia. A Glass Half Full? Gender in Migration Studies. International Migration Review, v. 40, n. 1, p. 3-26, 2006.

FUSASCHI, Michela. Quando il corpo è delle altre. Retoriche della pietà e umanitarismo-spettacolo. Torino: Bollati Boringhieri, 2011.

GREWAL, Inderpal; KAPLAN, Caren (a cura di). Scattered Hegemonies. Postmodernity and Transnational Feminist Practices. Minneapolis-London: University of Minessota Press, 1994.

HONDAGNEU-SOTELO, Pierrette. Gendered Transitions: Mexican Experiences of Immigration. Berkeley: University of California Press, 1994.

HOSKEN, Fran. Female Genital Mutilations and Human Rights. Feminist Issue, v. 1, n. 3, p. 3-24, 1981.

INDRA, Doreen M. Some Feminist Contributions to Refugees Studies. In: Gender Issues and Refugees: Development Implications and Crossing Boundaries: Exploring Knowledge, Power and Practice in Society (CASCA Annual Meetings). Toronto: York University, da 9 a 11 Maggio 1993.

LYNDSAY, Beverly. Comparative Perspectives of Third World Women: The Impact of Race, Sex and Class. New York: Praeger, 1983.

LUTZ, Helma. Gender in the Migratory Process. Journal of Ethnic and Migration Studies, v. 36, n. 10, p. 1647-1663, 2010.

MEHTA, Brinda. Postcolonial Feminism. In: CODE, Lorraine (a cura di). Encyclopedia of Feminist Theory. London: Routledge, 2000, p. 395-397.

McPHERSON, Kathryn. First wave/Second-wave feminism. In: CODE, Lorraine (a cura di). Encyclopedia of Feminist Theory. London: Routledge, 2000, p. 208-210.

PESSAR, Patricia R.; MAHLER, Sarah J. (a cura di). Transnational Migration: Bringing Gender. The International Migration Review, v. 37, n. 3, 2003.

PINELLI, Barbara. Migranti e rifugiate. Antropologia, genere e politica. Milano: Raffaello Cortina Editore, 2019.

PINELLI, Barbara. Salvare le rifugiate: gerarchie di razza e di genere nel controllo umanitario delle sfere di intimità. In: MATTALLUCCI, Claudia (a cura di). Antropologia e riproduzione. Attese, fratture e ricomposizioni della procreazione e della genitorialità in Italia. Milano: Raffello Cortina Editore, 2017, p. 155-186.

PINELLI, Barbara. Campi di accoglienza per richiedenti asilo. In: RICCIO, Bruno (a cura di). Antropologia e Migrazioni. Roma: CISU, 2014, p. 69-79.

PINELLI, Barbara. Migrare verso I'Italia. Violenza, discorsi, soggettività. Antropologia, n. 15, 2013, p. 7-20.

RIGO, Enrica. Donne attraversano il Mediterraneo. Una prospettiva di genere sulla protezione internazionale. Politeia, v. XXXII, n. 124, p. 82-94, 2016.

SALVATICI, Silvia. Introduzione. Genesis Profughe, v. III, n. 2, p. 5-20, 2004.

STARR, Chelsea. Third-wave feminism. In: CODE, Lorraine (a cura di). Encyclopedia of Feminist Theory. London: Routledge, 2000.

SNYDER, Claire. What Is Third-Wave Feminism? A New Direction Essay. Signs, v. 34 n. 1, p. 175-196, 2008 